\title{
Décadrages Décadrages
}

cinéma, à travers champs Cinéma, à travers champs

$43 \mid 2020$

Abdellatif Kechiche

\section{Vues imprenables sur la critique ou quand des journalistes écrivent leur propre histoire.}

Compte rendu de Philipp Brunner, Tereza Fischer, Marius Kuhn (éd.), Freie Sicht aufs Kino. Filmkritik in der Schweiz, Marburg, edition filmbulletin / Schüren, 2019, 176 p., env. 100 ill., noir et blanc

\section{Roland Cosandey}

\section{OpenEdition}

\section{Journals}

Édition électronique

URL : https://journals.openedition.org/decadrages/1605

DOI : 10.4000/decadrages. 1605

ISSN : 2297-5977

Éditeur

Association Décadrages

Édition imprimée

Date de publication : 1 juillet 2020

Pagination : 175-185

ISSN : 2235-7823

\section{Référence électronique}

Roland Cosandey, « Vues imprenables sur la critique ou quand des journalistes écrivent leur propre

histoire. », Décadrages [En ligne], 43 | 2020, mis en ligne le 15 octobre 2021, consulté le 06 mars 2022 URL : http://journals.openedition.org/decadrages/1605; DOI : https://doi.org/10.4000/decadrages. 1605 


\section{VUES IMPRENABLES SUR LA CRITIQUE OU QUAND DES JOURNALISTES ÉCRIVENT LEUR PROPRE HISTOIRE}

\author{
Compte rendu de Philipp Brunner, Tereza Fischer, Marius Kuhn (éd.), \\ Freie Sicht aufs Kino. Filmkritik in der Schweiz, \\ Marburg, edition filmbulletin / Schüren, 2019, \\ 176 p., env. 100 ill., noir et blanc
}

Freie Sicht aufs Kino: "freie Sicht", vue imprenable, dégagée d'obstacle, ou regard sans contrainte - le double sens renvoie à une vision particulière de la critique, celle qui ne porterait pas ce nom si elle ne s'exerçait en totale liberté.

L'exergue, emprunté au journaliste cinématographique Andreas Maurer, auteur de Filmriss! (2012), un ouvrage qui n'a pas franchi la barrière linguistique de ce pays plurilingue et dont aucun collègue romand ne semble avoir parlé ${ }^{1}$, met à cette condition première une injonction, qu'il énonce pour l'écriture, mais qui convient aussi pour la parole et l'image: "S'efforcer absolument à la plus grande précision possible ${ }^{2}$.

La perspective dessinée par l'avant-propos de ce recueil de textes ne dit pas autre chose quand on y pose une distinction entre critique de cinéma ("Filmpublizistik») et journalisme cinématographique ("Filmjournalismus») pour déplorer une réduction des espaces éditoriaux dévolus à la première depuis les années 1990 et la présence standardisée de la seconde entraînée par les mouvements de concentration de la presse écrite (p. 11).

Il faut donc comprendre que les éditeurs du recueil, Philipp Brunner, Tereza Fischer, Marius Kuhn, se réclament de cet espace, concret et symbolique, dont ils défendent l'esprit, un esprit caractérisé, nous disent-ils, par des qualités qui perdurent, même ailleurs que sur les supports de presse traditionnels, car heureusement "l'amour du cinéma conjugué à l'amour de l'écriture" fleurit toujours ${ }^{3}$. Ces deux passions, ils les exercent au sein de la revue filmbulletin, qui célèbre en 2019 son soixantième anniversaire par la présente publication.

Cette autoreprésentation valorisante et volontariste de la critique s'accompagne d'une vision sélective du passé. Tout en relevant que la première revue spécialisée suisse parut en 1911, sans préciser que Kinema était un corporatif lié au marché, l'avant-propos associe l'émergence de cette critique légitime à l'avant-garde cinématographique du muet. II en situe "l'âge d'or» dans les années 1970-1990,
Sauf erreur, à part les monographies de cinéastes publiées naguère par Pro Helvetia et l'Histoire du cinéma suisse 1896-1965 d'Hervé Dumont (1987), le seul ouvrage touchant au cinéma qui ait été édité dans les deux langues est la "chronique autobiographique" du cinéaste Rolf Lyssy, Swiss Paradise, paru en 2010, neuf ans après son édition originale. L'histoire des revues spécialisées montre que les assemblages linguistiques (français, allemand) furent le fait de deux catégories de périodiques, les corporatifs, liés aux associations des exploitants de salle et des loueurs, et une revue portée par la Fédération des ciné-clubs. Le seul périodique qui fasse aujourd'hui appel parfois à des signatures romandes publiées en français, cinema (Zurich), en est le lointain descendant (voir infra). Pour l'édition académique, les cas de bilinguisme éditorial partiel se comptent sur les doigts d'une main et sont rattachés à des recueils établis sous l'égide du Réseau cinéma $\mathrm{CH}$.

2 «Das Bemühen um grösstmögliche Präzision jedoch ist unabdingbar» (p. 5).

3 "die Liebe zum Film, gepaart mit der Liebe zu Schreiben » (p.9). 
$4 \quad$ En général, on se contente d'affirmer que le "vrai " critique, ce preux, "défend le cinéma" contre des obstacles variés, certains plus constants que d'autres: le moralisme et la censure, le mauvais goût et la paresse intellectuelle, et qu'il représente une sorte de guide. L'on s'accorde plus ou moins sur la difficulté de mesurer l'efficacité de cette action, sinon sur la vanité de l'exercice, et l'on déplore régulièrement le défaut de culture dont les autres critiques feraient preuve. On entend cela en filigrane de certains textes de ce recueil.

Pour la reconstruction de ce qui est une pensée du cinéma, on lira, à propos d'un critique lausannois, André Chaperon, "L'homme de nulle part. Frédéric-Philippe Amiguet, critique de cinéma", Revue historique vaudoise (Lausanne), n¹14, 1996, pp. 15-25, en ligne: https://www.e-periodica.ch; à l'échelle d'une problématique englobante, celle du rythme, on lira Laurent Guido, L'Âge du rythme. Cinéma, musicalité et culture du corps dans les théories cinématographiques françaises 19101930, Lausanne, I'Âge d'homme, 2007. En ligne: http://www.cinematheque.ch.

5 On prendra connaissance des conditions de la reconnaissance comme journaliste selon le Registre professionnel en allant à :

https://www.impressum.ch. c'est-à-dire à une période dont les rédacteurs peuvent se sentir les héritiers. Les années antérieures au milieu du $X X^{\mathrm{e}}$ siècle semblent appartenir à quelque âge obscur, bien que les publications d'Ernest Prodolliet, Fritz Güttinger, Laurent Guido, Gianni Haver, Pierre-Emmanuel Jaques, plus récemment d'Adrian Gerber, permettent de se faire une image moins rudimentaire d'un passé pas si lointain que ça.

Mais le journalisme vit de l'actualité et dans l'actualité, ses objets sont en constant renouvellement, la mémoire qu'on en a ne va guère au-delà de notre expérience de lecteur ordinaire. C'est ce qui explique qu'il est plus simple d'aborder la critique pour en énumérer le personnel, au fil du temps ou à telle période, ou de construire I'histoire d'une réception, qu'il s'agisse de Citizen Kane ou de Die Schweizermacher, du néo-réalisme ou du film gore, car les objets sont pour ainsi dire prédéfinis, que de chercher à reconstituer, à partir d'une multiplicité de textes de circonstance, ce que serait - pour tel et tel critique ou un groupe - une vision du cinéma ${ }^{4}$.

\section{2}

Plus ou moins explicitée, la vision qui se dégage de l'avant-propos et de la plupart des contributions de l'ouvrage nous permet de désigner un des objets de ce que pourrait être une véritable histoire de la critique: le discours que celle-ci porte sur elle-même quand il s'agit de justifier sa fonction. Que cette préoccupation, vieille comme la critique, qui en fait régulièrement l'exercice depuis le début du $x^{e}$ siècle dans notre domaine, ne se manifeste pas seulement comme un sujet, mais qu'elle apparaisse dans la production elle-même, quand on l'examine à la lumière de cette question, une seule étude en fait ici son propos, celle que Sylvain Portmann consacre à la fameuse émission de la Télévision suisse romande Spécial cinéma (1974-1993).

Son auteur doit probablement se demander ce que vient faire son étude parmi les autres contributions. Non pas que ces dernières seraient catégorie négligeable au nom de quelque hiérarchie du savoir, mais parce qu'en termes de questionnement et de méthodologie, l'ensemble ne relève pas de la discipline historique, même si des histoires il en est raconté, et de fort intéressantes au demeurant. L'utilité réflexive de l'étude de Portmann tient à ce qu'elle permet de définir le cadre épistémologique dans lequel Freie Sicht aufs Kino peut être appréhendé, celui d'une analyse générale de ce discours sur le cinéma que nous qualifierions volontiers de pragmatique, car il n'est ni de l'ordre de la théorie ni de l'ordre de l'historiographie. Ce n'est pas un objet homogène, unifié, mais un champ complexe, qui va, s'il faut parler en termes de producteur, de l'attaché de presse établissant des dossiers promotionnels au chroniqueur, journaliste RP ou pigiste ${ }^{5}$, multivalent ou spécialiste, en passant par le rédacteur de revue, de catalogue de festival, de programme de cinémathèque, ciné-club et autres entités culturelles.

Une telle perspective entraîne en premier lieu qu'un programme historiographique ne saurait reprendre à son compte la définition 
normative de l'activité critique telle qu'elle est formulée par les critiques eux-mêmes. Elle inclut aussi des acteurs qui, pour n'être pas des "critiques", n'en expriment pas moins définitions et valeurs: juristes, législateurs, censeurs, pédagogues, autorités morales ou intellectuelles, publicistes... Pour faire formule, disons que le terrain où se manifeste ce discours peut-être aussi bien le débat parlementaire, la presse généraliste que le trailer. Et il ne serait pas difficile de montrer comme cette variété de discours, d'émetteurs et d'intérêts de statut différent se caractérisent et interagissent, en prenant comme exemple les définitions du "cinéma suisse " formulées dès les années 1910, la discussion de la censure entre les années 1910 et 1980, en suivant la réflexion sur la loi fédérale du cinéma des années 1930 à 1960, ou encore en examinant comment fut traité le passage du cinéma analogique au cinéma numérique ${ }^{6}$.

En l'occurrence, le projet d'une histoire que nous avons sous les yeux provient de représentants de "la» critique, celle dont le genre premier est la recension des œuvres, sur le modèle littéraire, et son extension à d'autres protagonistes de la fabrique du cinéma. Le journalisme cinématographique, au sens de la production ou de la transmission d'une information sur ce domaine propre, constitue évidemment l'autre aspect de l'activité évoquée dans Freie Sicht aufs Kino. Relatée par des journalistes à la retraite ou en exercice, fondée sur le témoignage personnel, parfois sur des réminiscences et des opinions sollicitées auprès de collègues, sans doute aussi sur des archives personnelles, la relation de l'expérience professionnelle y tient souvent d'un "programme" plus ou moins explicite et peut valoir, dans certains cas, pour une manière de bilan.

3

Le sous-titre du recueil dit à son tour quelque chose: Filmkritik in der Schweiz. Nous le comprenons comme une volonté de représentativité. Un coup d'œil à la table des matières confirme cette préoccupation: une contribution est signée d'un auteur tessinois et porte sur le Tessin, trois émanent de Suisse romande, le reste est le fait de signatures principalement zurichoises et traite des manifestations de la critique en Suisse alémanique ${ }^{7}$.

Qu'en est-il de la périodisation? II y a un passé, qui remonte aux années 1950-1980, territorialement réparti entre trois témoins "historiques" entre-temps disparus, auxquels un écrit fut commandé en 1995, année où filmbulletin célébra, selon une convention bien établie, le centenaire du cinéma: Martin Schlappner (1919-1998), Freddy Buache (1924-2019), Guglielmo Volonterio (1926-2019).

II y a du contemporain, sous la forme d'une discussion sur la place des femmes et leur positionnement critique, menée entre Tereza Fischer, rédactrice en chef de filmbulletin depuis avril 2014, et Denise Bucher, alors rédactrice en chef de Frame, revue cinématographique de la NZZ am Sonntag ${ }^{9}$. D'aujourd'hui aussi, la présentation par Johannes Binotto de ce qu'il nomme un "format", I'essai-vidéo
$6 \quad$ Laurent Guido en fait la démontration dans une étude de cas: "Censeurs et cinéphiles. La critique de cinéma vue par les autorités de police lausannoises", Revue historique vaudoise (Lausanne), $\mathrm{n}^{\circ} 115,2007$, pp. 27-37. En ligne:

https://www.e-periodica.ch.

7 La critique de cinéma se manifeste soit à l'échelle de la région linguistique pour les périodiques, quelques journaux supra-cantonaux et les moyens radiophoniques et télévisuels, soit à celui des villes pour les autres quotidiens. Bien qu'elle réagisse à un marché national très homogène et aux mêmes grands festivals nationaux ou internationaux, sa perception est donc extrêmement parcellisée et l'établissement d'une vision globale un défi. Celui-ci pourrait commencer par l'étude de l'Association suisse des journalistes cinématographiques, créée en 1952, par celle de la représentation de la critique dans les commissions fédérales du cinéma, ou, pour les années antérieures, par l'examen de la mise en place juridique de l'autonomie constitutionnelle entre la partie rédactionnelle et la partie publicitaire des journaux et des résurgences récurrentes du conflit (Andreas Scheiner en mentionne quelques exemples dans son article).

8 Tereza Fischer a signé son dernier éditorial dans $n^{\circ} 1 / 2020$. À partir du numéro $2 / 2020$, la rédaction sera assumée par Selina Hangartner (elle aussi issue du Seminar für Filmwissenschaft) et Michael Kuratli.

Donnons une idée de cette livraison finale. Outre les recensions de films récents et les chroniques usuelles, le numéro (72 p.) propose trois articles thématiques unis par l'objet ‘animalı. Dans "Interspecies Rollenspiele. Anmerkungen zum Affenkino", Lukas Foerster traite de la représentation du singe au cinéma (pp.4-17, avec 18 généreuses illustrations); Vinzenz Hediger propose un court article intitulé "Der souveräne Herrscher der Weltmeer. 
Hans Haas und die Haie» (pp. 24-29, 6 ill.), consacré au biologiste marin autrichien Hans Haas (1919-2013), pionnier de la plongée et de la prise de vue subaquatique, et grand communicateur; Matthias Wittmann aborde la représentation de la pieuvre envisagée dans une perspective d'histoire culturelle avec "Out of Frame. Der Oktopus" (pp.60-67, 21 ill., en vignette) Notons une contribution (traduite) de Stéphane Gobbo, rédacteur culturel du Temps, sur la récente production de séries par la RTS en association avec des maisons de production indépendantes (p. 19). Publié depuis février 2014, en lien avec le Festival international du film de Zurich, le trimestriel Frame a paru pour la dernière fois le 8 décembre 2019 ( $\left.n^{\circ} 24\right)$. Son éditeur, NZZ am Sonntag, poursuit dès 2020 avec une

"Filmnewsletter» hebdomadaire multimédiale, dont Denise Bucher reste la rédactrice en chef.

10 "Das Videoessay als Zukunft der Filmkritik », pp. 157-172.

On peut prendre connaissance sur le site de filmbulletin des productions de Johannes Binotto et d'Oswald Iten:

https://www.filmbulletin.ch. L'article de Binotto est accessible en ligne:

https://www.zora.uzh.ch

11 "die unterschiedlichen Ausprägungsformen und [...] die sich veränderden Bedingungen für das Schreiben und Sprechen über Film in Schweizer Medien » (p. 9).

12 "eine facettenreicher Beitrag zur Geschichte der vielsprachigen Schweizer Filmkultur. "

13 "Der Sammelband ist ein erster unverzichtbarer Schritt in die Richtung einer überfälligen und umfassenden

Geschichte der Schweizer

Filmkritik. " (p. 11).

14 «Der Abdruck der drei nun ihrerseits historisch gewordenen Texte versteht sich nicht zuletzt als Anregung an jüngere Filmwissenschaftler und -wissenschaftlerinnen, sich donné très promotionnellement comme "l'avenir de la critique cinématographique ${ }^{10}$.

Au milieu vient un passé proche, dont le découpage varie, des années 1940 aux années 1980 pour la radio alémanique, 1970 à 1990 pour la Télévision suisse, les trente dernières années pour la presse écrite et l'émergence des sites internet.

Ces domaines d'intervention proposent judicieusement une troisième forme de représentativité, qui tient aux supports de la critique. À la presse généraliste et au périodique spécialisé (filmbulletin), viennent en effet s'ajouter la radio, la télévision, l'internet. C'est aborder, dans une ordonnance aussi chronologique que possible, "les différentes formes d'expression [...] et les conditions changeantes de la critique de cinéma écrite ou parlée dans les médias suisses. " ${ }^{11}$

On appréciera le bilinguisme discret mais effectif de la publication (en 1995, le texte de Freddy Buache avait été publié en allemand; celui de Volonterio est resté traduit). L'article de Stéphane Gobbo, chef de la rubrique culturelle du Temps, décrit la situation romande à partir de 1990, situe signatures et titres, souligne la réduction progressive, puis accélérée, de la presse d'opinion et d'information à une presse de communication, sans oublier de décrire l'émergence de canaux nouveaux apparus avec l'internet. Le tableau résulte d'une enquête menée auprès d'une douzaine de ses collègues. À «La critique en Suisse romande depuis 1990" (pp.87-100) fait pendant pour la Suisse alémanique "Untergang oder Übergang. Die Filmkritik im Digitalen Wandel " d'Andreas Scheiner (pp.67-86). De l'autre texte publié en français, l'analyse de l'émission Spécial cinéma, il sera question plus loin.

\section{4}

La visée du recueil est diversement formulée. La quatrième de couverture promet au lecteur "une contribution riche en facettes à l'histoire multilingue de la culture cinématographique suisse ". ${ }^{12}$

L'avant-propos est plus ambitieux: "Ce recueil constitue un indispensable premier pas vers une histoire attendue et détaillée de la critique cinématographique suisse. ${ }^{13}$ Chargé de situer la réédition des trois articles de 1995, Martin Girod conclut son introduction par ces mots:

Nous ne cacherons pas que la reprise de ces trois textes devenus entretemps historiques doit être entendue comme une incitation faite à la jeune génération des historiens et des historiennes à s'attacher à l'histoire de la critique cinématographique en Suisse, un domaine qui constitue un passionnant chapitre de I'histoire culturelle du $20^{\mathrm{ème}}$ siècle. Les contributions rassemblées ici sont un premier pas dans cette direction. Les matériaux transmis ne font pas défaut. ${ }^{14}$

Peu importe la qualification et ses nuances - multiplicité des facettes, contribution indispensable ou premiers éléments d'une histoire 
encore à faire -, l'ouvrage collectif publié chez Schüren (Marburg), se donne comme un apport à une histoire qui n'existe pas ou si peu, en quoi il est difficile de ne pas lui donner raison ${ }^{15}$. Initiative de l'une des deux plus anciennes revues suisses en activité, c'est aussi une défense et illustration de la critique de cinéma ${ }^{16}$.

5

Qui en sont les auteurs? La question n'est pas rhétorique. Elle cache une lacune à laquelle se heurte tout chercheur qui se lancerait par exemple dans une histoire de la réception en prenant la critique comme source: I'absence d'une approche prosopographique ${ }^{17}$. On peut esquisser quelques indicateurs en s'attachant non pas aux multiples noms mentionnés dans un texte qui ne se prête évidemment pas à l'exercice, mais aux auteurs rassemblés par le recueil lui-même.

Les notices qui leur sont consacrées en annexe nous renseignent avec une certaine inégalité dans le traitement. Ceux et celles pour qui la source est formalisée ou qui contrôlent leur propre image, se présentent selon une grille de données communes ${ }^{18}$, les autres sont traités assez sommairement (pp. 174-175).

Les douze personnes qui interviennent à des titres divers - éditeurs, auteurs, intervieweurs - se répartissent en trois générations bien distinctes que rapproche le fait qu'elles ont majoritairement une formation académique. Ceux qui sont nés entre 1969 et 1988 sont diplômés de l'Université de Zurich (le Seminar für Filmwissenchaft fut inauguré en automne 1989) ou de I'Université de Lausanne (la section Histoire et esthétique du cinéma débuta en automne 1990) ${ }^{19}$.

La deuxième génération compte trois contributeurs nés entre 1945 et 1951, retraités mais encore actifs dans leur domaine (Martin Walder a publié en 2017 la première monographie sur Goretta, Claude Goretta. Der empatische Blick). Ils sont tous passés par l'Université.

Curieusement, les notices se réduisent comme peau de chagrin pour les trois représentants de la génération la plus ancienne (qui est historiquement la troisième génération de critiques de cinéma en Suisse), dont la densité de l'intervention n'est guère traduite que par la longévité des interventions dans leur domaine respectif. Ses trois auteurs sont nés entre 1919 et 1926 et signaient en 1995 un de leurs derniers textes. Pour I'un d'eux, Martin Schlappner, on sait qu'il a le titre de docteur ès lettres, ayant défendu une thèse sur Thomas Mann und die französische Literatur en 1947.

En Suisse romande, aujourd'hui, deux critiques établis sont passés par Histoire et esthétique du cinéma, Stéphane Gobbo (diplômé en 2000) et Mathieu Loewer. Ils sont critiques professionnels depuis une quinzaine d'années. Les titulaires restent longtemps et les places sont chères, aussi est-ce ailleurs, dans des revues ou sur les sites en ligne que les diplômés de I'Université semblent trouver à s'exprimer, selon l'enquête de Gobbo (pp. 93-94).

Âge et formation ne sont que des indicateurs auxquels une recherche doit associer de nombreuses autres caractéristiques pour der Geschichte der Filmkritik in der Schweiz anzunehmen. Sie ist ein spannendes Kapitel der Geistesgeschichte im 20.Jahrhundert. Die in diesem Band versammelten Beiträge sind ein erster Schritt in diese Richtung - an überliefertem Material fehlt es nicht. " (p. 16).

15 La maison d'édition allemande Schüren (Marburg) occupe pour le Seminar für Filmwissenschaft de l'Université de Zurich la place de L'Âge d'homme (Lausanne) pour la section Histoire et esthétique du cinéma de I'Université de Lausanne. Publié par Chronos (Zurich) de 1995 à 2002, la collection Zürcher Filmstudien paraît chez Schüren depuis 2001, ainsi que les livres du Réseau Cinéma $\mathrm{CH}$. Deux revues suisses font partie du catalogue de la maison, filmbulletin et cinema dont le numéro 65 ("Skandal") est sorti en janvier 2020. Voir le catalogue en ligne:

https://www.schueren-verlag.de.

16 L'autre titre, c'est cinema, devenu annuel depuis 1983, précédé, sous le même titre par une publication trimestrielle (1961-1973), elle-même issue de Filmklub. Ciné-club (1955-1960).

Freie Sicht aufs Kino est la cinquième publication monographique des éditions filmbulletin. Les trois premières ont paru chez Lars Müller (Baden): Walter Ruggle, Theo Angelopoulos. Filmische Landschaft, 1990;

Sabine Brändli, Walter Ruggle, Bruno Jaeggi (éd.), Sowjetischer Film heute, 1990; Walter Ruggle (éd.), Nacer Khemir. Das verlorene Halsband der Taube, 1992. Le quatrième marque le début de la collaboration avec Schüren: Martin Walder, Claude Goretta - der empathische Blick, 2017. Merci à Tereza Fischer (filmbulletin) pour ces précisions. 
17 Les travaux d'Alessia Bottani sur les fondateurs des

Archives suisses du film (Bâle) et de la Cinémathèque suisse (Lausanne) montrent la fécondité d'une prosopographie qui dessine la configuration des protagonistes d'un groupe lié par les mêmes circonstances ou les mêmes visées:

http://wp.unil.ch.

18 Le site de filmbulletin est un bon exemple d'autoreprésentation de ce type (voir la rubrique "Autor_innen»sur la page d'accueil:

https://www.filmbulletin.ch). L'internet favorise cette personnalisation du critique, dont naguère seul le portrait photographique ornait à la rigueur la rubrique, en complément de la signature. Aujourd'hui, presse papier ou électronique, celle-ci est accompagnée d'un moyen d'accès direct à la personne: son adresse électronique.

19 Nous comptons Denise Bucher dans le nombre, car elle est moins interviewée qu'elle ne développe un propos amébée avec son interlocutrice. Comme son nom ne figure pas parmi les notices, précisons qu'elle est née en 1978 et que sa formation, études littéraires allemandes et "Filmwissenschaft», fut menée également à l'Université de Zurich. Sur Walter R. Vian, rédacteur en chef de filmbulletin de 1968 à 2014, lui aussi dépourvu de notice car objet d'un entretien, tout ce qu'on apprendra c'est qu'il se forma au cinéma dans le cadre du Katholischer Filmkreis Zürich, probablement aux débuts des années 1960 (pp. 58-59).

20 «Erkundung der Sprache des Films. Filmkritik in der französischen Schweiz», pp. 49-52. obtenir un tableau probant et des éléments aidant à la comparaison: statut journalistique, activité critique principale ou accessoire, mono ou polyvalence, lieu de publication et durée, fonction dans les associations ou les instances publiques subventionnantes en font nécessairement partie. La durée couplée à la nature de la publication est un indicateur important, dans la mesure où elle permet d'établir l'aire de rayonnement du critique et probablement d'avancer quelques indications objectives sur ce que l'on peut nommer son autorité.

\section{6}

Les auteurs du recueil proposent leur matière sous des formes diverses, parfois combinées: la réminiscence, l'interview, l'enquête, le dialogue, le tableau historique, l'étude de cas, la défense d'un genre. Le lecteur sensible à l'injonction de l'exergue appréciera la diversité des écritures, la variété des constructions et la singularité des voix.

Nous avons tout particulièrement goûté l'art de Martin Walder dans "Kino im Kopf. Das Deutschschweizer Radio und der Film» (pp.101-128). La contribution qu'il donne sur la critique radiophonique, dont il fut un des réalisateurs importants dans les années 1970-1990, conjugue avec un sens consommé du montage I'histoire de ce type d'émission depuis la fin des années 1930, des indications sur l'évolution des outils d'enregistrement et de la logistique des émissions, et des réflexions thématisées (la musique, les festivals, I'usage du dialecte ou du Hochdeutsch...). Cet ensemble propose ainsi une réflexion stimulante sur le "Radiofilm», défini comme l'art de faire voir des images avec des sons. La première vertu de ces pages est certainement de nous obliger à une attention formelle particulière, dont l'équivalent, pour l'étude d'une revue, serait la prise en compte de la composition graphique, de l'usage des images et de l'organisation des rubriques. Avec une différence qui est au cœur même de l'outil radiophonique tel que le définit Walder, sa proximité avec un matériau qu'il partage avec le cinéma, le temps.

Avec «Vom mählichen Werden eines Filmkritikers. Filmkritik in der Schweiz" (pp.18-39), Martin Schlappner est le seul de tous les critiques sollicités en 1995 comme en 2019 qui fasse état d'une connaissance historique de son métier, dans un texte où il tresse trois fils: sa propre formation à partir de sa passion cinéphilique de gymnasien, I'histoire de la critique en Suisse alémanique depuis les années 1910 (qui inclut celle de la Neue Zürcher Zeitung, où il était entré en 1950 et assuma de 1956 à 1984 la rédaction du "Feuilleton " culturel, intervenant dans plusieurs domaines: le cinéma, le tourisme, I'architecture, la gastronomie), et enfin le débat critique.

Là où Freddy Buache convoque une généalogie particulièrement sélective, par indifférence à l'Histoire ou par volontaire oubli, pour aboutir à l'affirmation plus ou moins implicite que la critique romande commence et finit par lui ${ }^{20}$, là où Guglielmo Volonterio, sans rien vraiment dire hélas de sa propre activité, peint un climat culturel marqué à ses yeux par une ignorance rédhibitoire et trouve malgré 
tout quelques noms à sauver ${ }^{21}$, Schlappner, lui, se décrit comme appartenant à une chaîne de critiques et entreprend de décrire les diverses positions intellectuelles de ses maillons, en lien avec deux sujets dont il a lui-même débattu, la relation entre cinéma et littérature et le cinéma suisse. Cette volonté de clarification intellectuelle est unique, non seulement dans ce recueil, mais d'une façon générale. Elle mériterait un examen qui tiendrait compte aussi des publications de I'auteur, omis par la notice, en particulier Von Rossellini zu Fellini: Das Menschenbild im italienischen Neo-Realismus (1958).

Dans sa conclusion, où il souligne les limites de son propre apport, Schlappner formule une expectation que Martin Girod exprimera à sa manière près d'un quart de siècle plus tard, dans son introduction: "Une histoire de la critique cinématographique? La seule réunion, pour commencer, de tout le matériel qui la constitue, remplirait un gros ouvrage. Les pistes indiquées ici demeurent modestes. Tout au plus signalent-elles l'ampleur de la tâche de I'historien, s'il s'en présente un. ${ }^{22}$

Nous partageons une telle attente, mais dans la perspective d'un travail collectif.

\section{7}

L'histoire des revues est une autre tache blanche dans l'historiographie suisse du cinéma et on peut espérer que les mises en ligne qui se poursuivent viendront stimuler la recherche (des taches blanches, I'histoire de l'exploitation et de la distribution, si étroitement liées à la critique, en sont également.... $)^{23}$. Aussi faut-il saluer l'entretien mené avec Walter Vian par Josef Stutzer, un des anciens collaborateurs de la revue filmbulletin, que Vian dirigea de 1968 à 2014 (" "Eigentlich gab es ja fast nichts". Ein Streitzug durch 60 Jahre Filmbulletin", pp. 53-66).

On regrettera toutefois que les réminiscences de Vian, qui évoque en grande partie les aspects techniques souvent négligés de la fabrication d'un périodique et les innovations qu'il apporta au sien, ne soient pas accompagnées de quelques précisions rédactionnelles et qu'on n'ait pas jugé bon de renseigner le lecteur, dans quelque annexe, sur le statut de la publication (qui est depuis 2013 une fondation), sur l'évolution de son lectorat et son aire de diffusion, sur l'origine de ses revenus (abonnements, vente au numéro, subventions) et la structure de son équipe (personnel technique, journalistes RP, pigistes) ${ }^{24}$.

Quand on lit les avanies de la critique à l'ère de la multimédialité digitale, telles que les expose Andreas Scheiner dans sa contribution chorale ("Untergang oder Übergang. Die Filmkritik im digitalen Wandel», pp.66-86), en particulier la drastique diminution du personnel critique (un quart des places supprimées ou réduites en 2008-2010), il n'est pas inutile de savoir concrètement comment filmbulletin peut continuer à s'enorgueillir du slogan "sechzig Jahre unabgängige Filmkritik in der Schweiz ${ }^{25}$. nia...". Filmkritik in der italienischen Schweiz", pp. 41-47. "Eine Geschichte der Filmkritik? Sie würde, wäre denn überhaupt erst all das Material erschlossen, das sie ausmacht, ein dickes Buch füllen. So bleiben wir denn bescheiden bei dieser Spurenlese. Welch einer Fülle der Historiker, gäbe es inn, begegnen würde, macht sie immerhin deutlich.» (p.39). ${ }^{23}$ Voir

https://www.e-periodica.ch $24 \quad$ Signalons que les années 2000-2019 de la revue sont accessibles en ligne sur son site et que les années antérieures y sont indexées. 

2016 près de $40 \%$ du budget provenait d'une subvention fédérale (p.86).

26 L'ouvrage n'a pas d'équivalent pour les cinquante dernières années. Rappelons que cette référence indispensable, à la fois bibliographie et anthologie, répertorie 53 périodiques parus entre 1911 et 1974, conservés en collections plus ou moins exhaustives par la Bibliothèque nationale (Berne). Sa "Chronologie der Filmpresse in der Schweiz" (pp.17-21) est trompeuse, si on oublie que la mesure est à cette aune. Nous estimons en effet que cet inventaire représente peut-être les $2 / 3$ des titres effectivement publiés durant la période envisagée, ce qui est d'ailleurs remarquable pour une bibliothèque qui n'est pas alimentée par le dépôt légal, si nationale soit-elle.

Manque ce qu'une définition extensive des modes de manifestation du discours sur le cinéma doit nécessairement inclure: les organes de liaison directe entre salles et spectateurs que sont les périodiques publiés par certains cinémas dès les années 1910, ephemera à peine visibles, car à peine conservés et de statut bibliothécaire fragile.
Les lecteurs de filmbulletin connaissent le soin que la revue porte aux images. S'agissant non pas de films, mais de ceux qui en parlent, on s'étonne à première vue de l'abondance iconographique du recueil, qui présente une centaine d'images en noir et blanc. Curieusement, en première et en seconde de couverture, I'habillage visuel prolonge ou complète le seul chapitre sur la radio, privilégiant l'exposition de diverses techniques de prise de son, dont il est effectivement question dans le texte de Martin Walder. Certaines photographies qui accompagnent l'entretien de l'ancien rédacteur en chef de filmbulletin semblent vouloir souligner l'aspect collectif du travail rédactionnel, mais par ailleurs le patchwork de couvertures plus ou moins recadrées ne documente pas vraiment l'évolution du graphisme de la revue.

II faut l'admettre avec un certain regret, le parti-pris de l'illustration est décoratif et on finit par ne plus prendre toutes ces images en compte. Sauf dans l'étude consacrée à Spécial cinéma, où le fourre-tout iconographique fait apparaître une incohérence manifeste entre cette indifférence à la valeur documentaire et démonstrative de l'image et les observations de l'analyse.

9

Quelques remarques à propos des sources que I'on rencontre au fil de la lecture. Disons d'abord qu'il aurait mieux valu ne pas produire de bibliographie plutôt que de bricoler une sélection de treize entrées en rassemblant paresseusement ce dont on avait une connaissance immédiate et superficielle (p. 175). La mention du précieux ouvrage d'Ernest Prodolliet, Die Filmpresse in der Schweiz (1975) nous permet de rappeler à quel point des outils de référence usuels dans d'autres disciplines font encore défaut dans la nôtre ${ }^{26}$.

Comme on peut le supposer d'après notre description de la nature des contributions - enquête contemporaine, entretien, souvenirs -, les archives ne sont pas des sources auxquelles on attendrait que l'on fasse nécessairement recours. Deux contributeurs y ont toutefois été puiser. Sylvain Portmann (le contraire eût été une contradiction dans le terme!), qui croise des sources diverses avec une grande efficacité, et Martin Walder, qui découvre les archives de ses prédécesseurs avec un plaisir presque jubilatoire et des résultats surprenants. Et de fait, les archives sonores et papiers de la SRF qu'il a explorées pour la part historique de sa relation promettent de telles richesses que I'on s'étonne de constater qu'elles n'aient pas encore été prises en compte comme une source majeure de la réception du cinéma suisse depuis la fin des années 1930.

Sylvain Portmann, dont la source première de l'analyse est visuelle (une sélection d'émissions de Spécial cinéma), outre I'hebdomadaire Radio-TV Je vois tout enfin accessible sur l'internet et des entretiens, met en garde sur un abus d'étiquette. Ce que la Télévision rend accessible aujourd'hui comme images, après "restauration", ce 
ne sont pas des documents d'archives au sens que l'on donne à ce terme dans le métier, mais des objets accommodés à une nouvelle circulation, sans que soient signalées les diverses altérations subies par les originaux.

Livrant des témoignages qui rendent compte d'une expérience et d'une histoire de la critique par les critiques eux-mêmes, produisant des aperçus inédits et des informations susceptibles de fournir plus d'un point de départ pour l'étude, qu'il s'agisse des trois textes de 1995 ou des contributions rassemblées en 2019, Freie Sicht aufs Kino offre un premier et précieux balisage de la critique de cinéma des années 1950 à nos jours. Si la valeur d'usage de l'ensemble aurait beaucoup gagné à la disposition d'un simple index des personnes et des titres de périodique, d'émissions et de sites, nous n'en souscrivons pas moins à la remarque de Martin Girod, qui voit dans ce recueil une occasion de stimuler la recherche historique, car la démonstration est faite: "Les matériaux transmis ne font pas défaut. ${ }^{27}$

ANNEXE

\section{«ÜBERLIEFERTES MATERIAL», UN PARCOURS DE SOURCES}

Il a été question plus haut des revues spécialisées, dont le nombre mis en ligne augmente peu à peu. Nous prolongeons ces indications par quelques repères, en privilégiant les ressources concernant principalement les personnes, sans prétendre à l'exhaustivité.

La source principale d'une histoire de la critique reste les articles eux-mêmes, parus dans la presse quotidienne et les périodiques auxquels l'accès sur l'internet est de plus en plus large. La mise à disposition prochaine des grands titres de la presse quotidienne genevoise facilitera grandement l'approche d'un des foyers critiques suisses les plus importants et cela depuis le début des années 1920.

\section{Autobiographies}

À tout seigneur, tout honneur, le récit établi par Freddy Buache (1924-2019) au fil des écrits et des entretiens forme le plus important corpus autobiographique dont on dispose à propos d'un critique, en l'occurrence un protagoniste qui fut avant tout, à partir de l'autorité que lui conféra pendant près d'un demi-siècle sa position comme directeur de la Cinémathèque suisse, un publiciste au sens ancien du terme. Les entretiens publiés sous le titre Derrière l'écran (1995) en constituent la forme la plus achevée.

Par ailleurs, les écrits autobiographiques ne sont pas nombreux: Viktor Zwicky (1891 ?-1968), So war es damals.

Aus den Memoiren eines Zeitungsreporters (1965), Luigi Caglio (1899-1982), I/ spettatore anziano (1965), Christian Defaye (1934-1997), Tant qu'il y aura des étoiles (1999), Marcel Leiser (1945), "Marcel Leiser, profession: cinéphile» (2012, Documents de cinéma de la Cinémathèque suisse).

L'enquête orale Cinémémoire relevant du récit de vie, on mentionnera, parmi les 21 entretiens suisses romands, quatre critiques: Freddy Buache, Bruno Edera (1937), Freddy Landry (1930-2019), Marcel Leiser. Voir http://wp.unil.ch/cinememoire.

Parmi les 40 entretiens suisses alémaniques, on en compte quatre aussi, avec des protagonistes aux fonctions plus composites: Max Peter Amman (1929), Alex Bänninger (1942), Franz Ulrich (1936), Mario Gerteis (1937-2016), Philippe Dériaz (1930).

Voir https://wp.unil.ch/cinememoire/volet-alemanique-du-projet. 
Natalie Fritz, Charles Martig, Fabian Perlini-Pfister (éd.), Nur für reife Erwachsene. Katholische Filmarbeit in der Schweiz, Zurich, Theologischer Verlag, 2011. Adrian Gerber, "Eine gediegene Aufklärung und Führung in dieser Materie»: Katholische Filmarbeit in der Schweiz 1908-1972, Fribourg (CH), Academic Press, 2010 (Religion, Politik, Gesellschaft in der Schweiz, $n^{\circ}$ 53).
Travaux monographiques

Trois critiques ont fait l'objet d'un mémoire de licence ou de master: Maurice Porta (1879-1941), Eva Elie (1892-1969), Freddy Buache (1924-2019).

Leur titre en indique l'axe thématique et la périodisation:

Laurent Guido, Maurice Porta à la Feuille d'Avis de Lausanne et les débuts de la critique cinématographique en Suisse romande, 1920-1932, Université de Lausanne, 1997;

Andrea Rusconi, "La Cinémathèque c'est moi! " Freddy Buache e la Cinémathèque suisse (1948-1975). Progetti culturali e dibattiti ideologici, Université de Fribourg, 2007;

Adèle Morerod, "Va pour les autres!" Eva Elie, une voix critique en marge (1923-1929), Université de Lausanne, 2019.

Les deux premiers sont inédits, le troisième est accessible en ligne: https://serval.unil.ch/notice/serval:BIB_S_28590

Le critique de cinéma est souvent un rédacteur culturel polyvalent, ce qui est illustré par l'activité d'un Frank Jotterand (1923-2000): Daniel Vuataz, "Toutes fenêtres ouvertes", Franck Jotterand et la Gazette littéraire. Deux décennies d'engagement culturel en Suisse romande (1949-1972), 2012;

d'un Manuel Gasser (1909-1979): David Streiff, Manue/ Gasser. Biographie, 2016;

ou d'un Martin Schlappner (1919-1998): Martin Schlappner, Journalismus aus Leidenschaft: Medien, Architektur, Tourismus. Standpunkte und Perspektiven (Alex Bänninger, Baltz Livio, Jakob Zweifel (éd.), 1989).

Recueil d'articles

Ce dernier ouvrage, qui est une anthologie, permet de rappeler que les recueils d'articles installent dans la durée la production du critique, de son vivant ou à titre posthume, et constituent une importante marque de reconnaissance.

Pour la Suisse, cette rubrique comporte à peine une poignée de noms en un siècle. Un auteur occupe quantitativement une place unique, Freddy Buache, pour avoir publié, comme il le rappelle dans son article (p.51), "mehr als dreissig [...] Bücher» issus de son activité critique, à L'Âge d'homme, une maison d'édition où il dirigeait la collection cinéma.

Mentionnons deux autres titres. De Fritz Güttinger (1907-1992)، spécialiste de littérature anglo-américaine et de cinéma muet, ses articles parus dans la Neue Zürcher Zeitung: Köpfen Sie mal ein Ei in Zeitlupe! Streifzüge durch die Welt des Stummfilms, 1992; du cinéaste Alexander J. Seiler (1928-2018), collaborateur de la Weltwoche et du Tages-Anzeiger, dont les archives sont déposées à la Cinémathèque suisse: Daneben geschrieben 1958-2007, 2008.

Archives

Les fonds papier de la Cinémathèque suisse conservent quelques ensembles de documents rattachés à des critiques, des revues ou des institutions. Parmi les plus importants, mentionnons les fonds Freddy Buache, Bruno Edera, Marcel Leiser, Marcel Schüpbach et les papiers de Freddy Landry, en cours de versement au moment où nous écrivons, sans oublier les fonds de l'Office protestant du cinéma et du périodique Ciné-Feuilles.

L'antenne zurichoise de l'institution, la Dokumentationsstelle, a été établie sur I'héritage archivistique de l'importante activité critique de I'Eglise catholique et de l'Eglise évangélique, dont on méconnaît ou, pire, dont on déconsidère, malgré deux travaux publiés sur l'action des catholiques $^{28}$, le rôle effectif et évolutif qu'elle a joué entre 1940 et 1980 et cela bien au-delà du cercle confessionnel. La Dokumentationsstelle s'est 
enrichie d'apports ultérieurs comme les archives de Martin Schaub (1937-2003) ou celles de l'Association suisse des journalistes cinématographiques (ASJC)/Schweizerischer Verband der Filmjournalistinnen und Filmjournalisten (SVFJ), fondée en 1952. Les fonds papier inventoriés de la Cinémathèque sont décrits en ligne: https://caspar.cinematheque.ch.

Les dossiers documentaires de la Cinémathèque suisse sont largement constitués d'articles correspondant à leur objet (réalisateur, film, thèmes), ce qui en fait les sédiments d'une activité critique suisse romande et française depuis les années 1950: voir https://www.cinematheque.ch/f/ actualites/article/dossiers-documentaires-premiers-inventaires-en-ligne.

Le Seminar für Filmwissenschaft (Zurich) conserve un fonds Martin Schlappner (1919-1998) et un fonds Felix Bucher (1938-1989);

la section Histoire et esthétique du cinéma (Lausanne) un fonds André Ehrler (1900-1949) que complète le fonds André Ehrler des Archives d'État de Genève ${ }^{29}$.
29 Merci à Caroline Neeser, Seraina Winzeler (Cinémathèque suisse), Pierre-Emmanuel Jaques (Université de Lausanne) et Martin Girod (Zurich) pour leurs informations. 